\title{
Author Correction: STEEP mediates STING ER exit and activation of signaling
}

\section{Bao-cun Zhang, Ramya Nandakumar, Line S. Reinert (1), Jinrong Huang, Anders Laustsen, Zong-liang Gao (D),} Cheng-long Sun, Søren Beck Jensen, Anne Troldborg @i, Sonia Assil, Martin F. Berthelsen, Carsten Scavenius (D,

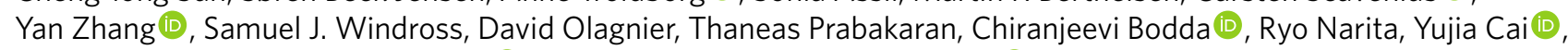
Cong-gang Zhang, Harald Stenmark (D), Christine M. Doucet, Takeshi Noda (D), Zheng Guo, Raphaela Goldbach-Mansky,

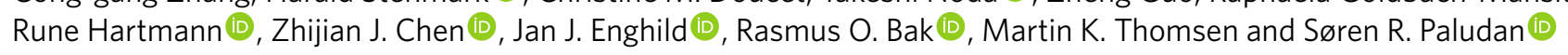

\section{Correction to: Nature Immunology https://doi.org/10.1038/s41590-020-0730-5, published online 20 July 2020.}

In the version of this article initially published, there were a number of errors in the figures. In Fig. $2 \mathrm{~d}$, the treatment on the right of the graph was incorrectly labeled as "dsDNA." The cells were treated with cGAMP. In Fig. 3d, the immunoblot probe was incorrectly labeled as an anti-phosphorylated-STING antibody (" $\alpha$-pSTING"). The correct label is " $\alpha$-STING." In Fig. $5 d$, the confocal microscopy images showing THP-1 cells stained with an anti-STING antibody were omitted, and the treatment for the bottom row of images was incorrectly labeled as "cGAMP." The cells were treated with PtdIns(3)P. The correct Fig. 5d is shown below. In Fig. 5e, the antibody used for the immunoblot in the bottom row was incorrectly labeled as anti-vinculin. The antibody used was anti-STEEP. In Fig. 6b, the labels for the cGAMP treatment time did not line up with the correct lanes of the immunoblots and some labels were omitted. The correct time labels (in $\mathrm{h}$ ) for both the ER fraction and the cell lysate are, from left to right, $0,1,0$, 1. In Extended Data Fig. 5h, the confocal microscopy images of the mock-treated STEEP knockout THP-1 cells duplicated the images of the mut5 STEEP mRNA-rescued cells. The correct Extended Data Fig. 5h is shown below. The errors have been corrected in the HTML and PDF versions of the article.
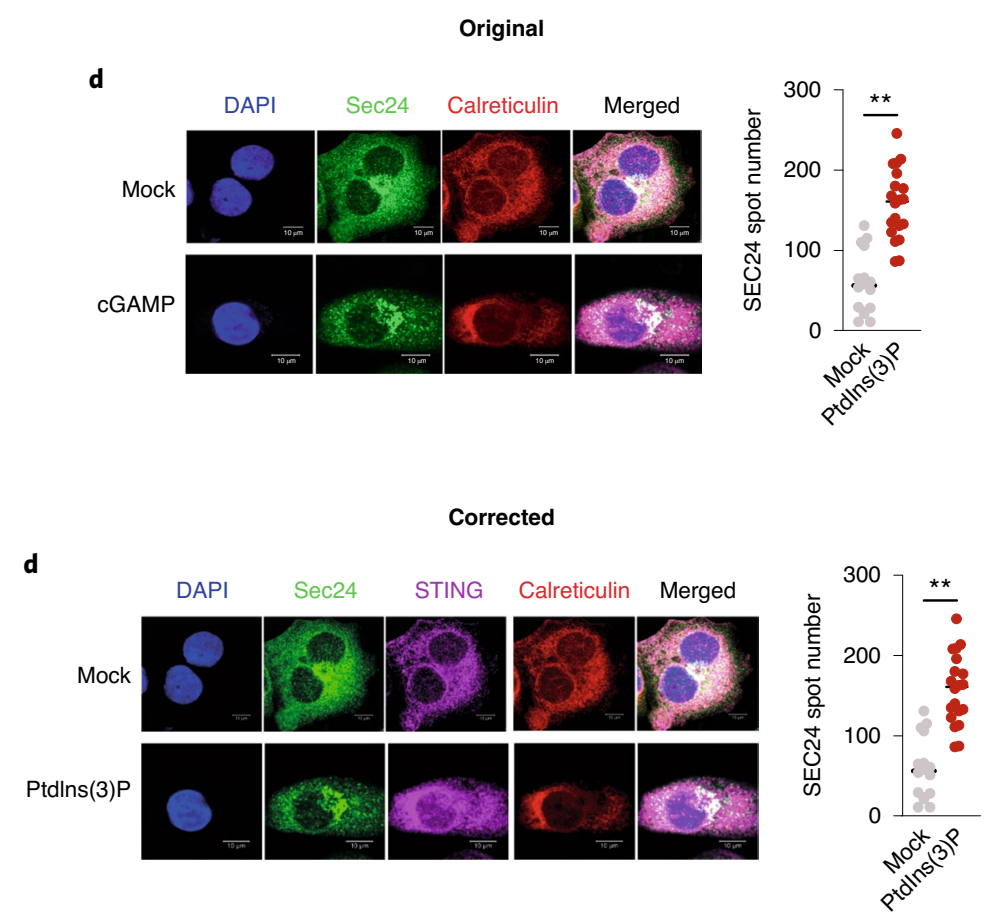

Fig. 5 | Original and Corrected. 


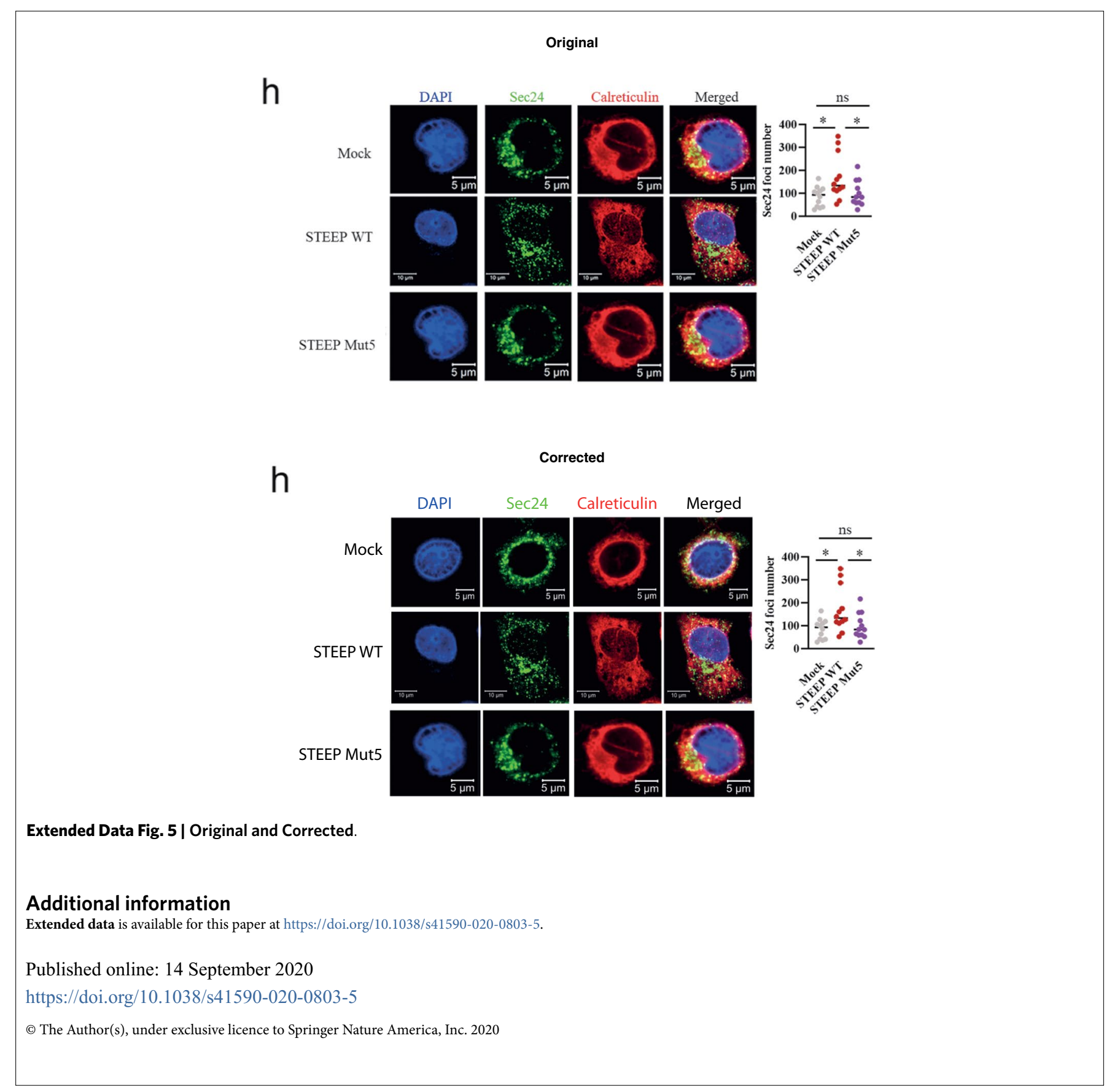

